\title{
Fitting the Long-Range Order of a Decagonal Quasicrystal
}

\author{
M. Chodyń, P. Kuczera And J. Wolny \\ Faculty of Physics and Applied Computer Science, AGH University of Science and Technology, \\ al. A. Mickiewicza 30, 30-059 Krakow, Poland
}

\begin{abstract}
The generalized Penrose tiling is an infinite set of decagonal tilings. It is constructed with the same rhombs (thick and thin) as the conventional Penrose tiling, but its long-range order depends on the so-called shift parameter $s \in\langle 0,1)$. The formula for structure factor, calculated within the average unit cell approach, works in physical space only and is directly dependent on the $s$ parameter. It allows to straightforwardly change the long-range order of the refined structure just by changing the $s$ parameter and keeping the tile decoration unchanged. The possibility and viability of using the shift as one of the refinement parameters during structure refinement was tested for a numerically generated simple binary decagonal quasicrystal.
\end{abstract}

DOI: 10.12693/APhysPolA.130.845

PACS/topics: $61.44 . \mathrm{Br}$

\section{Introduction}

The structure analysis of decagonal quasicrystals (DQCs) is still a great challenge. One of the important problems is determining the long-range order (LRO) of the structure, i.e. the right kind of decagonal tiling. Current models, namely, the higher dimensional $(\mathrm{nD})$ approach $[1,2]$ and the average unit cell (AUC) approach [3-6] share common problem, they assume the type of tiling during first step of model building. A vast majority of (if not all) models of DQCs in the literature are based on the Penrose mutual local derivability (PMLD) class of tilings [7] e.g. pentagonal Penrose tiling, rhombic Penrose tiling, hexagon-star-boat tiling. The LRO for all PMLD class tilings is the same and if the structure is described by one tiling from the PMLD class it can also be described by any other tiling from this class. This gives us the possibility to choose the tiling that is most convenient to describe structural details. Even though PMLD class of tilings was successfully used to describe various DQC phases, it is still an open question if PMLD class tilings really describe their LRO in the best way.

PT can be easily generalized to the so-called generalized Penrose tiling (GPT) $[8,9]$ which, in principle, does not belong to the PMLD class. The unit tiles of GPT are the same two Penrose rhombs as in PT, hoverer the matching rules are different. This results in different possible tile arrangement. The kind of obtained tiling depends on a certain continuous parameter called shift $s \in\langle 0,1)$. Therefore there is an infinite set of different GPTs for different $s$ values, and each of them has a different LRO. The diffraction properties of GPT have been studied before, e.g. [10], also the structure factor of arbitrarily decorated GPT in the AUC approach has been calculated $[6,11]$. The formula works in physical space only and is a function of the parameter $s$. This allows to change the LRO of the structural model with fixed unit tile decorations. The goal of this paper is to show how the GPT concept can be practically applied for the refinement process of DQCs.

\section{Structure factor}

As a base for our calculation we will use the structure factor of an arbitrarily decorated GPT. The derivation of Eq. (1) has been shown in [6,11], from where we will rewrite it

$$
\begin{aligned}
F & =\sum_{T=\{L, S\}} \sum_{f=1}^{3} \sum_{\theta=1}^{5} \exp \left(\mathrm{i} \varphi\left(z_{\perp}^{A}\right)\right) \int_{\operatorname{AUC}\left(T_{f, \theta}\right)} \\
& \times \exp \left(\mathrm{i}\left(\boldsymbol{\kappa}_{\boldsymbol{n}, \boldsymbol{m}} \cdot \boldsymbol{u}\right)\right) \mathrm{d} u \sum_{j}^{n_{T_{f, \theta}}} p_{j} f_{a}^{j} \exp \left(\mathrm{i} \boldsymbol{k}_{\boldsymbol{n}, \boldsymbol{m}} \boldsymbol{r}_{j}^{T_{\theta}}\right),
\end{aligned}
$$

where

$$
\begin{aligned}
& \varphi\left(z_{\perp}^{A}\right)=-\frac{4 \pi}{5}\left[\left(n_{1}+n_{2}\right)-0.5\left(m_{1}+m_{2}\right)\right] z_{\perp}^{A}, \\
& \kappa_{x}=\frac{2 \pi}{5}\left[\left(n_{1}+n_{2}\right)-\tau\left(m_{1}+m_{2}\right)\right], \\
& \kappa_{y}=\frac{2 \pi}{5} \tau \sqrt{\tau+2}\left[\left(n_{1}-n_{2}\right)-\tau\left(m_{1}-m_{2}\right)\right], \\
& k_{x}=\frac{2 \pi}{5 a}\left[\left(n_{1}+n_{2}\right)+\frac{\left(m_{1}+m_{2}\right)}{\tau}\right] \\
& k_{y}=\frac{2 \pi}{5 a} \tau \sqrt{\tau+2}\left[\left(n_{1}-n_{2}\right)+\frac{\left(m_{1}-m_{2}\right)}{\tau}\right] .
\end{aligned}
$$

First sum runs over types of rhombuses (thick and thin), second sum runs over possible rhombs families, third sum over possible orientations of a given unit and forth sum run over atoms decorating a given unit in a given orientation. $\boldsymbol{k}_{\boldsymbol{n}, \boldsymbol{m}}$ is parallel component of reciprocal lattice vector given by indices of $n_{1}, n_{2}, m_{1}, m_{2} . \boldsymbol{r}_{j}^{T_{\theta}}$ is the position of the $j$-th atom in a given structure unit in a given orientation, $f_{a}^{j}$ - the atomic scattering factor of a given atom and $p_{j}$ is the fraction of an atom inside the structure unit.

The formula in Eq. (1) requires, however, minor modifications if we want to use it in structure refinement 
algorithm. First, we need to add the Debye-Waller factors (also called the atomic displacement parameter, ADP), both phononic (Eq. (2)) and phasonic (Eq. (3)):

$$
\begin{aligned}
& D_{j}\left(\boldsymbol{k}_{\boldsymbol{n}, \boldsymbol{m}, h_{5}}\right)= \\
& \quad \exp \left(-\frac{1}{16 \pi^{2}}\left[\left(k_{x}^{2}+k_{y}^{2}\right) b_{x y}^{j}+k_{z}^{2} b_{z z}^{j}\right]\right) .
\end{aligned}
$$

The ADP is related to the thermal vibrations of the atoms: $b_{x y}$ is proportional to the squared average amplitude of the thermal vibrations in the quasiperiodic plane and $b_{z z}$ is proportional to the squared average amplitude of the thermal vibrations in the periodic direction.

$$
D_{\mathrm{Ph}}\left(\boldsymbol{k}_{\boldsymbol{n}, \boldsymbol{m}}^{\perp}\right)=\exp \left(-\frac{1}{16 \pi^{2}}\left(\kappa_{x}^{2}+\kappa_{y}^{2}\right) b_{\mathrm{Ph}}\right) .
$$

$D_{j}\left(\boldsymbol{k}_{\boldsymbol{n}, \boldsymbol{m}}^{\perp}\right)$ is related to the phasonic flips, which are typical for quasicrystalline structures [12]. The parameter is characteristic for the whole structure and is proportional to the average amplitude of phason flips squared.

The values of atomic scattering factors were calculated using Eq. (4). The parameter values $a_{i} b_{i} c$ were taken from crystallographic tables [13] for the atoms used in the simulation

$$
f\left(\frac{\sin \theta}{\lambda}\right)=\sum_{i=1}^{4} a_{i} \exp \left(-b_{i} \frac{\sin ^{2} \theta}{\lambda^{2}}\right)+c .
$$

The final formula of structure factor that is used in the refinement algorithm is presented in Eq. (5):

$$
\begin{aligned}
& F=D_{\mathrm{Ph}}\left(\boldsymbol{k}_{\boldsymbol{n}, \boldsymbol{m}}^{\perp}\right) \sum_{T=\{L, S\}} \sum_{f=1}^{3} \sum_{\theta=1}^{5} \exp \left(\mathrm{i} \varphi\left(z_{\perp}^{A}\right)\right) \\
& \times \int_{\operatorname{AUC}\left(T_{f, \theta}\right)} \exp \left(\mathrm{i}\left(\boldsymbol{\kappa}_{\boldsymbol{n}, \boldsymbol{m}} \cdot u\right)\right) \mathrm{d} u \\
& \times \sum_{j}^{n_{T_{f, \theta}}} p_{j} D_{j}\left(\boldsymbol{k}_{\boldsymbol{n}, \boldsymbol{m}, h_{5}}\right) f_{a}^{j} \exp \left(\mathrm{i} \boldsymbol{k}_{\boldsymbol{n}, \boldsymbol{m}} \boldsymbol{r}_{j}^{T_{\theta}}\right) .
\end{aligned}
$$

\section{Shift refinement}

To test the viability of refinement of the shift parameter, we simulated a simple binary quasicrystal with arbitrary decoration. It was built using two atomic species: $\mathrm{Al}$ (blue dots) and $\mathrm{Rh}$ (red dots). The building units are shown in Fig. 1. Formula (5) was used to calculate a reference diffraction pattern (shift parameter of GPT was equal to $s=0.234$ ) (Fig. 2).

After obtaining the reference diffraction pattern, which was used as an "experimental data set" we run two different tests. First test was to prepare plot of $R$-factor (Eq. (6)) as function of shift, to see if and how the $R$ factor changes with changing $s$. The results are presented in Fig. 3. We can see two minima, one very strong, corresponding to the exact value of the shift parameter $(s=0.234)$ in the reference structure. The second one is located symmetrically at position $s=0.766$. It is much weaker due to lack of symmetry of the decoration around the center of the thick rhomb. If a symmetric decoration

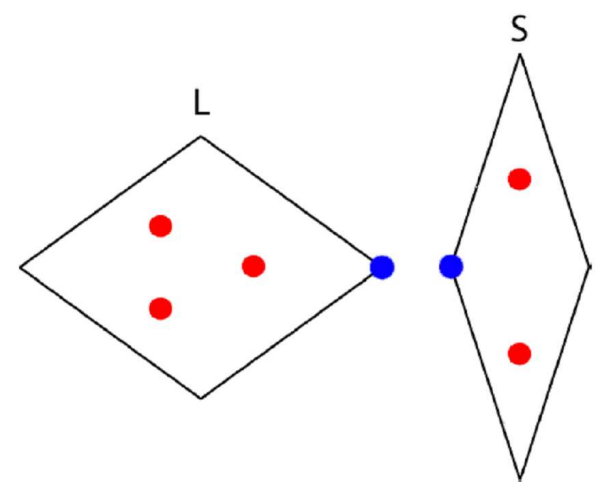

Fig. 1. Building units, with decoration used in refinement procedure.

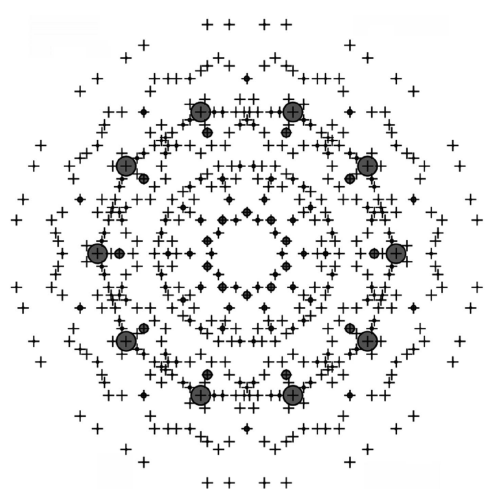

Fig. 2. Diffraction pattern used as reference in refinement procedure, crosses indicate position of peaks that were too small for a circle to be drawn.

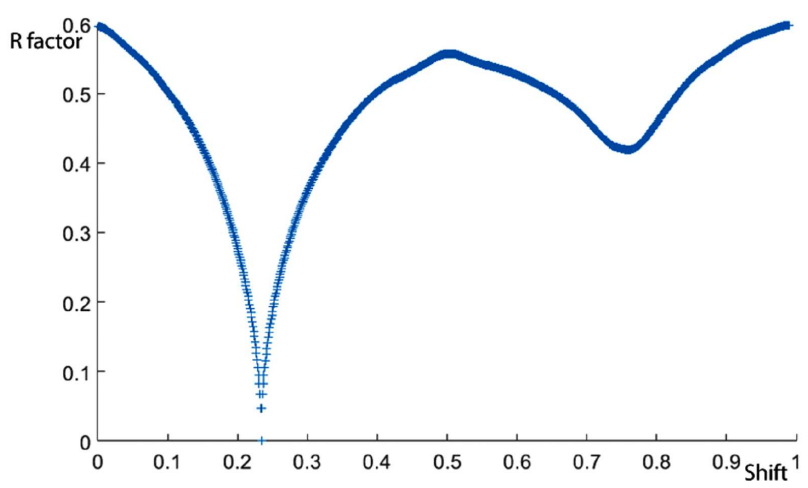

Fig. 3. $R$-factor in function of shift, calculated for the GPT $s=0.234$ with simple decoration.

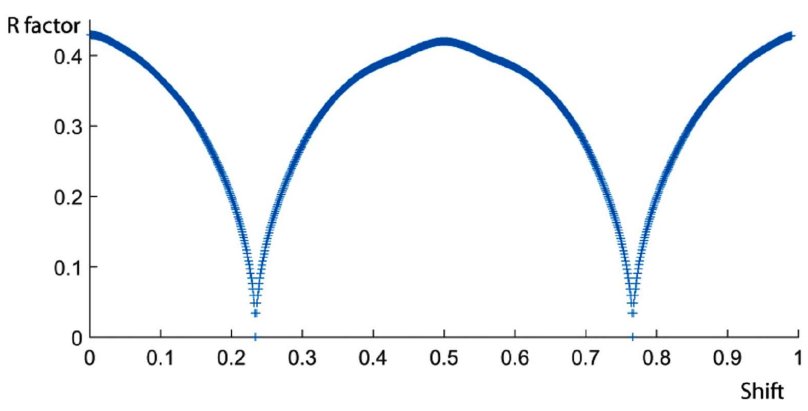

Fig. 4. As in Fig. 3, but with no decoration. 
was used, both minima would be equally strong (Fig. 4):

$$
R\left(F^{2}\right)=\frac{\sum\left|F_{\mathrm{obs}}^{2}-F_{\text {calc }}^{2}\right|}{\sum\left|F_{\mathrm{obs}}^{2}\right|} .
$$

Second test was to run the refinement procedure using shift as one of the fit parameters. It is relatively easy to find the correct shift value, even in case where the starting values of other parameters (e.g. atom positions, or ADP factors) did not match exactly the ones in the reference structure. However we have to remember that there are two possible solutions and avoid getting stuck in the weaker minimum. We also noticed that while it is possible to run simultaneously refinement of shift with other parameters and obtaining correct values, it is recommended to run shift parameter refinement separately from the rest, as it increases the required number of iterations tremendously.

\section{Conclusions}

We have shown an example of a structure refinement that used the shift as one of the fit parameters. The refinement was done for a simple binary decagonal quasicrystal simulated numerically. We have shown that two different minima can be found, usually one being much stronger than the other. The difference in the minimum's strength is related to the symmetry of the decoration. After successful test of the viability of using the shift in refinement for simple quasicrystal, we believe it is possible to run such procedure for a real quasicrystal. Using one of preliminary solved and refined structures, like $\mathrm{Al}-$ $\mathrm{Cu}-\mathrm{Rh}$ DQC [14], would be good starting point for making an attempt to find the right GPT for this structure, which would imply the refinement of LRO.

\section{Acknowledgments}

Authors kindly acknowledge financial support from National Science Center, Poland, under grants no. DEC2013/11/B/ST3/03787

\section{References}

[1] P.M. De Wolff, Acta Crystallogr. A 30, 777 (1974).

[2] T. Janssen, Acta Crystallogr. A 42, 261 (1986).

[3] J. Wolny, Acta Crystallogr. A 54, 1014 (1998).

[4] J. Wolny, B. Kozakowski, P. Repetowicz, J. Alloys Comp. 342, 198 (2002).

[5] A. Dabrowska, B. Kozakowski, J. Wolny, Acta Crystallogr. A 61, 350 (2005).

[6] B. Kozakowski, J. Wolny, Acta Crystallogr. A 66, 489 (2010).

[7] M. Baake, M. Schlottmann, P.D. Jarvis, J. Phys. A Math. Gen. 24, 4637 (1991).

[8] A. Pavlovitch, M. Kleman, J. Phys. A Math. Gen. 20, 687 (1987).

[9] K.N. Ishihara, A. Yamamoto, Acta Crystallogr. A 44, 508 (1988).

[10] M.V. Jaric, Phys. Rev. B 34, 4685 (1986).

[11] M. Chodyn, P. Kuczera, J. Wolny, Acta Crystallogr. A 71, 161 (2015).

[12] A. Yamamoto, Acta Crystallogr. A 52, 509 (1996).

[13] International Tables For Crystallography, Volume $C$, Mathematical, physical and chemical tables, Ed. E. Prince, 2006.

[14] P. Kuczera, J. Wolny, W. Steurer, Acta Crystallogr. B 68, 578 (2012). 